\title{
Supernova progenitor mass constraints through spatial correlations with host galaxy star formation
}

\author{
Joseph P Anderson \\ Departamento de Astronomía, Universidad de Chile, Casilla 36-D, Santiago, Chile \\ email: anderson@das.uchile.cl
}

\begin{abstract}
We present progenitor mass constraints on supernovae ( $\mathrm{SNe})$, from correlations with star-forming regions within host galaxies. Through a pixel statistics method used together with $\mathrm{H}$-alpha imaging of host galaxies, we present a progenitor mass sequence running from supernovae type Ia (SNIa) arising from the lowest, through SNII, SNIb, and finally SNIc arising from the highest mass progenitors, implied from an increasing association of their explosion sites with star formation (SF). We also present constraints on the various core-collapse (CC) sub-types, finding the perhaps surprising result that SNIIn show the lowest degree of association with SF of any CC type, implying relatively low-mass progenitors. Finally, we compare the SNIIn environment distribution to that of SNIa, posing the provocative question that additional SNIIn may be linked to the SNIa phenomenon where the latter's spectra are hidden beneath that of circumstellar material (CSM) interaction.
\end{abstract}

Keywords. (stars:) supernovae: general, (ISM:) HII regions

\section{Introduction}

Constraining SN progenitor characteristics through observing their immediate environments within host galaxies has become a strong area of research in recent years. This approach, consisting of investigating the nature of stellar populations in close proximity to the explosion sites of SNe enables one to build statistically significant samples of events, while also using the characteristics of stellar populations most representative of that of their progenitors for obtaining constraints.

To date these investigations have involved two distinct approaches. The first involves using imaging of nearby SN host galaxies and has often used some form of pixel statistics to measure the degree of association of different SN types with different types of (usually young) stellar populations (see e.g. Leloudas et al. 2010; Kelly \& Kirshner 2012). This has led to various constraints on differences in the ages of environments of CC SNe, and hence progenitor mass constraints. The second approach consists of obtaining spectra of host HII regions of SNe within galaxies, which can be used to investigate the environment metallicities of different SN types. This approach has led to a series of CC SN progenitor metallicity constraints, in particular constraints on differences between the different SN types which may explain differences in their transient evolution (Modjaz et al. 2008; Anderson et al. 2010; Leloudas et al. 2011; Modjaz et al. 2011; Sanders et al. 2012; Stoll et al. 2012).

In these proceedings we summarise the most recent results to arise from an investigation of the association of different SN types with SF within galaxies, using SN host galaxy imaging and pixel statistics. 


\section{Host galaxy pixel statistics}

Over the last decade we have obtained a large sample of $\mathrm{H} \alpha$ imaging of SN host galaxies. $\mathrm{H} \alpha$ line emission within galaxies traces on-going $\mathrm{SF}$, as the emission is a result of the recombination of ISM hydrogen ionised by the UV flux of massive stars. Therefore one can use this narrow-band emission as a tracer of the distribution of massive stars within galaxies, and hence use this to probe differences in the massive star environments of different types of SNe. Within our current sample (published in Anderson et al. 2012) we have a sample of host galaxy imaging for: $163.5 \mathrm{SNII}$; which can be separated into 58 IIP, 13 IIL, 13.5 IIb, 19 IIn and 12 'impostors', plus 48 with no sub-type classification, and $96.5 \mathrm{SNIbc} ; 39.5 \mathrm{Ib}, 52 \mathrm{Ic}$ and 5 with no sub-type classification. In addition we have a sample of 98 SNIa which we are analysing, comparing environment characteristics with light-curve properties (Anderson et al. in prep.).

To analyse these data we use a pixel statistics method first outlined in James \& Anderson (2006), then further described in Anderson \& James (2008). This method produces a value between 0 and 1 for every pixel (an 'NCR' value) of the continuum-subtracted $\mathrm{H} \alpha$ image, where 0 indicates a pixel consistent with sky values or zero emission flux, while

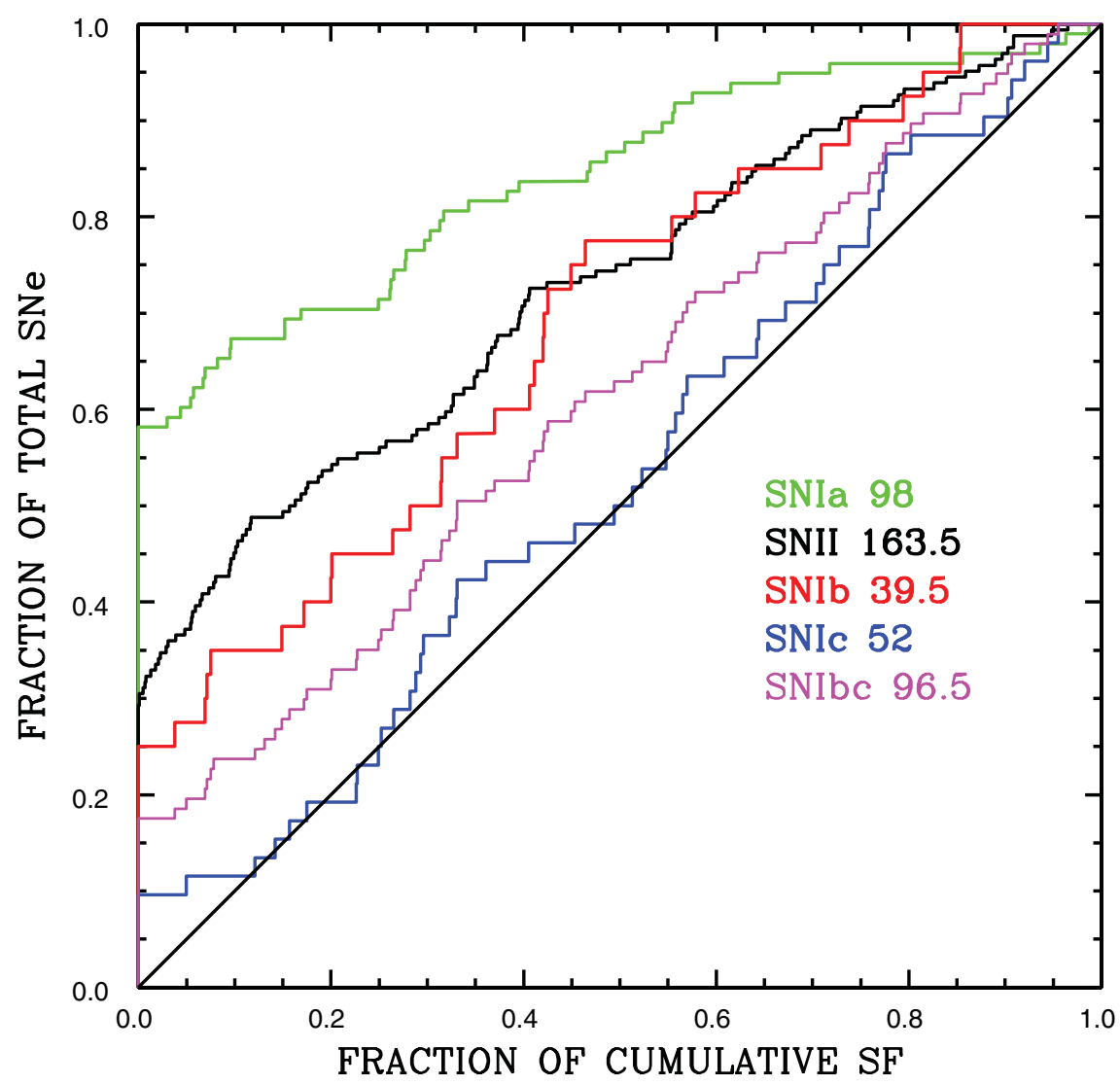

Figure 1. Cumulative distributions of the NCR statistics for the main SN types: SNIa in green, SNII in black, SNIb in red, SNIc in blue, and the overall SNIbc population in magenta. The diagonal black line shows a hypothetical population which shows a one-to-one correlation with the $\mathrm{H} \alpha$ emission within host galaxies. As a distribution moves away from this population to the upper left of the plot, the SN population is showing a lower degree of association to the emission. 
1 indicates the pixel has the highest count of any pixel within the image. Using this statistic, distributions for different SN types can be built. The statistic is formalised in such a manner that if the $\mathrm{H} \alpha$ count within a pixel is directly proportional to the amount of SF occurring within that region of the galaxy, and a $\mathrm{SN}$ population directly traces the SF within their host galaxies, then we expect a flat distribution of NCR values for that population, with a mean NCR value of 0.5 . Following this, these distributions are analysed and used to provide progenitor constraints: if a population has a lower mean NCR value then it is assumed that this relates to on average longer stellar lifetimes, and lower progenitor masses, implied from the lower association to the on-going SF.

\section{Progenitor constraints}

In figure 1 the NCR distributions for different SN types are presented. These are plotted cumulatively: as distributions move away to the top left of the plot from the diagonal black line, they are displaying a progressively lower degree of association with the ongoing $\mathrm{SF}$ as traced by $\mathrm{H} \alpha$, which implies longer stellar lifetimes and lower progenitor masses. In fig. 1 we see a clear implied sequence of progenitor masses, starting with the SNIa showing the lowest correlation with SF, and therefore having the lowest progenitor masses (as expected), through the SNII, the SNIb and finally the SNIc showing the highest correlation with host galaxy HII regions, therefore arising from the highest mass progenitors. While the SNII and SNIb distributions are quite similar, with the SNIb showing a slightly higher HII region association than the SNII, the SNIc are statistically significantly separated from SNIb: this implies a difference in the mean lifetimes and hence masses between these two types.

In fig. 2 the distributions of various SN sub-types are presented. The most surprising result to arise from these statistics would appear to be that the SNIIn population shows one of the lowest degrees of association with the SF, indeed showing a similar (but slightly lower) correlation with host galaxy HII regions as the SNIIP. This is surprising because the progenitors of SNIIn are often claimed to be Luminous Blue Variable stars (LBVs) (e.g. Smith 2008). However, LBVs are thought to be very massive stars which one would expect to be associated with on-going SF. Hence, in contrast our results imply
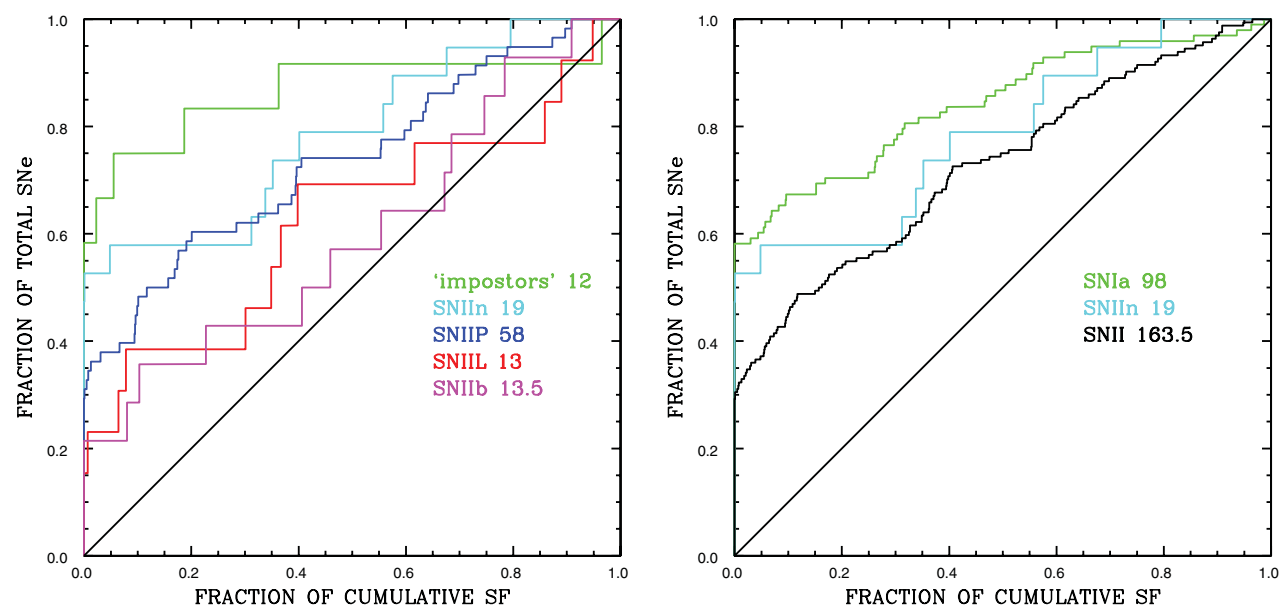

Figure 2. Cumulative distributions of NCR statistics for SN sub-types. Left: Comparison of SNII sub-types: 'impostors' in green, SNIIn in cyan, SNIIP in blue, SNIIL in red, and SNIIb in magenta. Right: Comparison of the SNIa (green), SNIIn (cyan) and overall SNII (black) distributions. 
that at least the majority of SNIIn arise from relatively low mass progenitors. This is also consistent with Kelly \& Kirshner (2012), who found similar environment properties for SNIIn and SNIIP. It is also noted that in the left panel of fig. 2 there appears to be some evidence that SNIIL and SNIIb are more highly correlated with the line emission than SNIIP, possibly suggesting higher mass progenitors for the former.

Finally, in the right panel of fig. 2 a comparison is made between the SNIIn, SNIa and SNII NCR distributions. The fact that the SNIIn distribution lies between the other two populations is intriguing. A number of SNIa have been reported to explode within a high density CSM environment (see Hamuy et al. 2003; Dilday et al. 2012). Therefore, one may speculate that there are additional SNIa where the CSM interaction is sufficiently strong to hide characteristic SNIa features, hence these are classified as SNIIn. If this speculation is true then this would naturally explain the low degree of association of SNIIn with on-going SF.

\section{Conclusions}

SN environmental studies can provide useful constraints on progenitor differences between different SN types. Using pixel statistics and $\mathrm{H} \alpha$ pixel statistics a mass sequence has been proposed for the main SN types running SNIa-SNII-SNIb-SNIc, in terms of increasing progenitor mass implied from an increasing association to host galaxy HII regions. In addition, it has been found that the SNIIn show a relatively low degree of association with the line emission, a result which is inconsistent with the majority of their progenitors being LBV stars, but which could be consistent with the hypothesis that additional SNIIn have SNIa-like progenitors, where the spectra are dominated by features of CSM interaction rather than those from the thermonuclear runaway.

Acknowledgments: J.A. acknowledges support from FONDECYT grant 3110142, and grant ICM P10-064-F (Millennium Center for Supernova Science), with input from 'Fondo de Innovacin para la Competitividad, del Ministerio de Economa, Fomento y Turismo de Chile'.

\section{References}

Anderson, J. P. \& James, P. A., 2008 MNRAS, 390, 1527

Anderson, J. P., et al. 2010 MNRAS, 407, 2660

Anderson, J. P., et al. 2012 MNRAS, 4241372

Dilday, B., et al. 2012 Science, 337, 942

Hamuy, M., et al. 2003 Nature, 424, 651

James, P. A. \& Anderson, J. P., 2006 A $\mathcal{G} A, 453,57$

Kelly, P. L. \& Kirshner, R. P., 2011 ApJ, 759, 107

Leloudas, G., et al. $2010 A \mathscr{E} A, 518,29$

Leloudas, G., et al. 2011 A $\mathcal{E} A, 530,95$

Modjaz, M., et al. 2008 AJ, 136, 1136

Modjaz, M., et al. 2011 ApJ, 731, 4

Sanders, N. E., et al. 2012 ApJ, 758, 132

Smith, N. 2008 IAUS 250, 193

Stoll, R., et al. 2012 arXiv 1205.2338

\section{Discussion}

CAPpellaro: Are you worried of a possible bias for classification of type IIn in particular when they occur in the middle of bright HII regions? 
ANDERSON: Indeed this is a worry. We are currently compiling all available data on the SNIIn in our sample to look at these possible selection effects.

Koo: I wonder if you can discriminate whether SNe that you observe belong to the current generation of stars or to the previous generation of stars.

Anderson: There is no way of knowing which episode of SF the SN is related to. However, as stated in my talk, our method is completely statistical in nature: we would not claim that any individual SN is young or old dependent on an association. We only claim differences between $\mathrm{SNe}$ in a statistical sense.

WANG: Is it possible to establish the association on a more probability base?

Anderson: Yes, such approach has been adopted for association of SNe with galaxy types. It may also been done for HII regions based on the separation of a SN from an $\mathrm{H}$ alpha-emitting region. 